Pesq. Vet. Bras. 29(8):632-636, agosto 2009

\title{
Dosagem de marcadores cardíacos CK-MB e Tnlc e eletrólitos no envenenamento experimental por veneno de sapo em cães ${ }^{1}$
}

\begin{abstract}
Annelise C. Camplesi ${ }^{2}$, Michiko Sakate ${ }^{2}$, Natália M.B. Simão² e Carla F. Moya ${ }^{3}$
ABSTRACT.- Camplesi A.C., Sakate M., Simão N.M.B. \& Moya C.F. 2009. [Dosing cardiac markers CK-MB and Tnlc and electrolytes in experimental toad envenoming in dogs.] Dosagem de marcadores cardíacos CK-MB e Tnlc e eletrólitos no envenenamento experimental por veneno de sapo em cães. Pesquisa Veterinária Brasileira 29(8):632-636. Departamento de Clínica Médica Veterinária, Faculdade de Medicina Veterinária e Zootecnia, Universidade Estadual Paulista, Botucatu, SP 18600-000, Brazil. E-mail: annecamplesi@yahoo.com.br

Among the systemic signs of toad venom (bufotoxin) poisoning in dogs, the cardiotoxic effects are one of the most important. Thus, the objective of this experiment was to evaluate potential changes in the cardiac muscle in dogs poisoned experimentally by toad venom and to observe the eletrolyte alterations which may occur in this condition. Twenty dogs divided into control group $(n=5)$ and poisoned group $(n=15)$ were utilized. The toad venom was extracted by manual compression of the paratoidic glands. After general anesthesia, dogs in the control group received placebo and dogs in the poisoned group received the venom by orogastric catheter. Samples for dosage were collected 6 hours and 24 hours after poisoning and 0, 2, 4, 6 and 12 hours after poisoning for electrolytes dosage. The Man-Withney test was used for statistical analysis $(P<0.05)$. The poisoned dogs showed (saline) elevated levels of cardiac markers CK-MB and Tnlc, confirming the cardiotoxic effect of the bufotoxin. Hypokalemia and hypocalcemia were also observed.
\end{abstract}

INDEX TERMS: Toad venom poisoning, bufotoxin, cardiac markers, electrolyte, dogs.

RESUMO-- Dentre os sinais sistêmicos causados pelo envenenamento por veneno de sapo (bufotoxina) em cães, os efeitos cardiotóxicos são um dos mais importantes. $O$ objetivo deste estudo foi avaliar as potenciais alterações no músculo cardíaco de cães envenenados experimentalmente por veneno de sapo e observar as alterações eletrolíticas que podem ocorrer nesse tipo de envenenamento. Utilizaram-se 20 cães divididos em grupo controle $(n=5)$ e grupo envenenado $(n=15)$. O veneno de sapo foi extraído por meio de compressão manual das glândulas paratóides. Após anestesia geral, os cães do grupo con-

\footnotetext{
${ }^{1}$ Recebido em 27 de janeiro de 2009.

Aceito para publicação em 12 de março de 2009.

2 Departamento de Clínica Médica Veterinária, Faculdade de Medicina Veterinária e Zootecnia (FMVZ), Universidade Estadual Paulista (Unesp), Botucatu, SP 18600-000, Brasil. *Autor para correspondência: annecamplesi@yahoo.com.br

${ }^{3}$ Departamento de Reprodução Animal e Radiologia Veterinária, FMVZ-Unesp, Botucatu, SP.
}

trole receberam placebo (solução fisiológica) e os do grupo envenenado uma alíquota do veneno por sonda orogástrica. As colheitas de sangue para dosagem dos marcadores cardíacos foram realizadas seis e 24 horas após o envenenamento. As colheitas de sangue para dosagem dos eletrólitos foram realizadas antes e duas, quatro, seis e 12 horas após o envenenamento. A análise estatística empregada foi o teste não-paramétrico de Mann-Withney $(P<0,05)$. Os cães envenenados por veneno de sapo apresentaram elevação dos níveis dos marcadores cardíacos CK-MB e Tnlc, confirmando a cardiotoxicidade do veneno. Hipocalemia e hipocalcemia foram também observadas nos cães envenenados.

TERMOS DE INDEXAÇÃO: Veneno de sapo, bufotoxina, marcadores cardíacos, eletrólitos, cães.

\section{INTRODUÇÃO}

Muitas espécies de anfíbios possuem glândulas secretoras cutâneas que previnem o ressecamento da pele e os pro- 
tegem dos predadores. Os sapos, quando atacados por outros animais, secretam substâncias por essas glândulas. Essas secreções têm efeito citotóxico e podem causar hemólise (Klaassen 1996). Sapos do gênero Bufo têm distribuição mundial e predileção pelas áreas de clima tropical ou temperado úmido (Monti \& Cardello 1994). Existem várias espécies no Brasil, como $B$. ictericus, $B$. schneideri, B. rufus e B. marinus (Zelnik 1965, Bedford 1974). Os cães são as vítimas mais comuns desse tipo de envenenamento, pois são atraídos pelos movimentos lentos dos sapos, principalmente no período noturno (MacDonald 1990). Cães envenenam-se pela ingestão ou pelo abocanhamento do sapo, o que provoca o contato e a absorção do veneno entre a mucosa oral ou do trato digestório (Knowles 1968, Monti \& Cardello 1994, McFarland 1999).

O veneno de Bufo spp. possui dois grandes grupos de substâncias ativas: as aminas biogênicas e os derivados esteróides (Zelnik 1965). Das aminas biogênicas são destacadas, pela importância toxicológica, adrenalina, noradrenalina, serotonina, bufoteninas, di-hidrobufoteninas e bufotioninas. Dos derivados esteróides, destacamse os bufodienólides e as bufotoxinas, que agem de forma semelhante aos digitálicos, causando a inibição da bomba de sódio e potássio das células da musculatura cardíaca (Zelnik 1965, Chen \& Kovarikova 1967, Hoffman \& Lefkowitz 1991, Eubig 2001).

Os sinais clínicos do envenenamento por veneno de sapo são divididos em três grupos, de acordo com a gravidade: leve, moderado ou grave. Em casos leves, há irritação da mucosa oral e sialorréia. Quadros moderados apresentam, além de irritação da mucosa oral e sialorréia, êmese, depressão, fraqueza, ataxia, sinais neurológicos, anormalidades do ritmo cardíaco, evacuação e micção espontâneas. Em casos graves, pode haver ainda diarréia, dor abdominal, midríase não-responsiva, convulsões, edema pulmonar e cianose. As alterações eletrocardiográficas observadas são arritmias, que podem evoluir para taquicardia ventricular multiforme e, se o cão não for tratado, para fibrilação ventricular e morte (Knowles 1968, Otani et al. 1969, Bedford 1974, Palumbo et al. 1975, Russel 1979, Palumbo \& Perry 1983, Oliveira 1998, McFarland 1999, Sakate \& Oliveira 2000, 2001).

Alguns autores (Sonne et al. 2008) relataram o óbito de um cão Bulldog Francês por envenenamento por veneno de sapo. Neste relato, o cão foi encontrado morto pelo proprietário, sem sinais clínicos anteriores, e a causa morte foi confirmada por análise toxicológica através da cromatografia em camada delgada direcionada para alcalóides e da cromatografia gasosa, baseada no método Stas-Otto.

Uma forma de avaliar a função cardíaca é a dosagem de marcadores cardíacos. Essas substâncias são capazes de indicar lesão das células cardíacas ou o nível de ativação dos sistemas compensatórios. Dentre os marcadores cardíacos são importantes a creatinaquinase fração MB (CK-MB), a mioglobina, as troponinas, a aspartato aminotransferase e a lactato desidrogenase (Ettinger
1997). A CK-MB e a troponina I - fração cardíaca (Tnlc) são os marcadores mais específicos e os mais indicados para verificar lesões agudas no miocárdio (Missov et al. 1997, Puschendorf 1999).

Este estudo teve como objetivo verificar se cães envenenados por veneno de sapo apresentam alterações celulares no músculo cardíaco, por meio da dosagem de CK-MB e Tnlc, e descrever as alterações eletrolíticas observadas nesse tipo de acidente.

\section{MATERIAL E MÉTODOS}

$O$ veneno foi colhido de 20 sapos da espécie Bufo schineideri, provenientes do Laboratório de Herpetologia do Departamento de Zoologia do Instituto de Biociências, Universidade Estadual Paulista (UNESP), Campus de Botucatu. O anfíbio foi colocado no interior de uma caixa de isopor parcialmente tampada com um vidro e a extração se realizou comprimindo a glândula paratóide na direção do vidro. Devido à viscosidade, o veneno aderia ao vidro e então era removido com auxílio de uma espátula. Um pool de veneno foi armazenado em frascos de vidro e encaminhado ao Laboratório de Imunologia, Departamento de Microbiologia e Imunologia, Instituto de Biociências, Unesp, Campus de Botucatu, onde foi realizada a liofilização em liofilizador convencional (Edwards do Brasil, Liobras Ind. Com. e Serv. de Liofilizadores Ltda, Av. Comendador Alfredo Maffei 3879, Jardim São Carlos, São Carlos, SP) a $-30^{\circ} \mathrm{C}$, durante três dias. Após esse procedimento, o veneno foi dividido em alíquotas iguais e equivalentes a $70 \%$ da quantidade de veneno de um sapo; tais alíquotas foram acondicionadas em tubo de ensaio de $20 \mathrm{~mL}$ e conservado a $5^{\circ} \mathrm{C}$.

Utilizaram-se 20 cães sem raça definida, machos e fêmeas, adultos, hígidos, com aproximadamente $10 \mathrm{~kg}$ cada e provenientes do Biotério Central da Unesp, Campus de Botucatu. Os cães foram divididos em grupos controle $(n=5)$ e envenenado $(n=15)$. Após jejum de 12 horas, os cães foram submetidos à indução anestésica direta com tiopental sódico, $25 \mathrm{mg} / \mathrm{kg}$, intravascular (Tiopenta|®, Cristália Produtos Químicos Farmacêuticos Ltda, Rodovia Itapira-Lindóia, Km 14, Itapira, SP) e manutenção anestésica com isoflurano a $3 \%$ (Isoflurano ${ }^{\circledR}$, Cristália Produtos Químicos Farmacêuticos Ltda, Rodovia Itapira-Lindóia, Km 14, Itapira, SP). Durante todo o período anestésico, os cães foram mantidos em colchão térmico para controle da temperatura corpórea. Foi utilizada a ventilação controlada em todos os cães ( $F R=10-15 \mathrm{mpm})$.

Para o envenenamento experimental, as alíquotas de veneno foram ressuspensas em $10 \mathrm{~mL}$ de água e administradas a cada animal por sonda orogástrica. Os cães do grupo controle receberam $10 \mathrm{~mL}$ de solução fisiológica.

Colheu-se o sangue dos cães nos tempos T0 (antes da administração do veneno), T1 (duas horas após administração do veneno), T2 (quatro horas após administração), T3 (seis horas após administração), T4 (12 horas após administração) e T5 (24 horas após administração do veneno). As dosagens de CK-MB, com reativo seco (Vitros, Ortho-Clinical Diagnostics, 100 Indigo Creik Dr, Rochester, NY, USA), e de Tnlc, com reagente (Flex Troponina I Cardíaca, Dimension, Dade Behring, Ludwig-Erhard-Str. 12, Eschborn, Germany), para avaliar as possíveis lesões no músculo cardíaco, foram realizadas nos tempos T3 e T5. A hemogasometria (Stat Profile pH Ox Plus L, Megalab, Av. das Américas 700/214B, Barra da Tijuca, Rio de Janeiro, RJ) para dosagem plasmática de sódio $\left(\mathrm{Na}^{+}\right)$, cálcio 
iônico $\left(\mathrm{Ca}^{++}\right)$e potássio $(\mathrm{K}+)$, utilizou amostras de sangue arterial colhidas com seringa heparinizada, por punção da artéria safena, nos períodos T0, T1, T2, T3 e T4.

A análise estatística empregada para avaliar a variável CKMB e os eletrólitos foi a multivariada de Perfil (Morrison 1990) e para a variável Tnlc foi o teste não-paramétrico de MannWithney (Zar 1996). O nível de significância adotado foi de 5\% de probabilidade.

\section{RESULTADOS}

Os valores da CK-MB dos cães do grupo controle variaram de $0,107 \mathrm{ng} / \mathrm{mL}$ a $0,446 \mathrm{ng} / \mathrm{mL}$, com média de $0,360 \mathrm{ng} / \mathrm{mL}$ em T3 e média de $0,175 \mathrm{ng} / \mathrm{mL}$ em T5. Os cães envenenados experimentalmente apresentaram valores de CK-MB entre $0,02 \mathrm{ng} / \mathrm{mL}$ e $0,755 \mathrm{ng} / \mathrm{mL}$, com média de $0,543 \mathrm{ng} /$ $\mathrm{mL}$ em T3 e média de $0,185 \mathrm{ng} / \mathrm{mL}$ em T5. Observou-se diferença significativa entre os grupos no momento T3, pois os cães envenenados apresentaram média de CK-MB significativamente maior que a média do grupo controle nesse momento $(\mathrm{P}<0,05)$, como mostra o Quadro 1.

Os valores de Tnlc dos cães do grupo controle variaram de $0,08 \mathrm{ng} / \mathrm{mL}$ a $0,16 \mathrm{ng} / \mathrm{mL}$, com média de $0,11 \mathrm{ng} /$ $\mathrm{mL}$ em T3 e média de $0,08 \mathrm{ng} / \mathrm{mL}$ em T5. Os cães envenenados experimentalmente apresentaram valores de Tnlc entre $0,05 \mathrm{ng} / \mathrm{mL}$ e $2,47 \mathrm{ng} / \mathrm{mL}$, com média igual a $0,12 \mathrm{ng} /$ $\mathrm{mL}$ em T3 e média de $0,26 \mathrm{ng} / \mathrm{mL}$ em T5. Diferença significativa foi detectada entre os grupos no momento T5 $(P<0,05)$, conforme verificado no Quadro 2.

Quadro 1. Valores médios e desvio padrão de CK-MB, em $\mathrm{ng} / \mathrm{mL}$, dos cães dos grupos controle $(n=5)$ e intoxicado por veneno de sapo $(n=15)$, nos dois momentos avaliados

\begin{tabular}{ccc}
\hline Grupos & T3 $(6$ horas $)$ & T5 $(24$ horas $)$ \\
\hline Controle & $0,360 \pm 0,086^{\mathrm{a}}$ & $0,175 \pm 0,039^{\mathrm{a}}$ \\
Intoxicados & $0,543 \pm 0,169^{\mathrm{b}}$ & $0,185 \pm 0,099^{\mathrm{a}}$ \\
Valor $\mathrm{P}$ & $(\mathrm{P}=0,033)$ & $(\mathrm{P}=0,822)$
\end{tabular}

Letras iguais na mesma coluna não diferem entre si $(P<0,05)$.
Quadro 2. Valores médios e percentuais (P25; P75) de Tnlc, em ng/mL, dos cães dos grupos controle $(n=5)$ e intoxicado com veneno de sapo $(n=15)$, nos dois momentos avaliados

\begin{tabular}{cccccccc}
\hline \multirow{2}{*}{ Grupos } & \multicolumn{3}{c}{ T3 (6 horas) } & & \multicolumn{3}{c}{ T5 (24 horas) } \\
\cline { 2 - 3 } \cline { 6 - 7 } & Mediana & P25 & P75 & & Mediana & P25 & P75 \\
\hline Controle & $0,11^{\mathrm{a}}$ & 0,09 & 0,138 & & $0,08^{\mathrm{a}}$ & 0,08 & 0,095 \\
Intoxicados & $0,12^{\mathrm{a}}$ & 0,1 & 0,255 & & $0,26^{\mathrm{b}}$ & 0,14 & 0,347 \\
Valor $\mathrm{P}$ & $(\mathrm{P}=0,407)$ & - & - & & $(\mathrm{P}=0,005)$ & - & -
\end{tabular}

Letras iguais na mesma coluna não diferem entre si $(P<0,05)$.

Os valores de sódio plasmático no grupo controle variaram de $140 \mathrm{mEq} / \mathrm{L}$ a $149 \mathrm{mEq} / \mathrm{L}$, e todos permaneceram dentro da normalidade (DiBartola 2000). No grupo envenenado, os valores de sódio plasmático variaram entre 133mEq/L e 150mEq/L; 73\% (11/15) dos cães apresentaram valores levemente abaixo dos valores de referência para a espécie; $27 \%$ (4/15) apresentaram valores dentro da normalidade. Nenhum animal apresentou hipernatremia e não houve diferença significativa entre os momentos e nem entre os grupos $(P>0,05)$.

No grupo controle, os valores de potássio plasmático variaram de $2,9 \mathrm{mEq} / \mathrm{L}$ a $4,2 \mathrm{mEq} / \mathrm{L}$, e todos os cães desse grupo apresentaram valores levemente abaixo dos normais (DiBartola 2000). No grupo envenenado, os valores variaram de $2,5 \mathrm{mEq} / \mathrm{L}$ a $5,9 \mathrm{mEq} / \mathrm{L}$ e, da mesma forma, os cães apresentaram valores levemente abaixo dos de referência para a espécie; apenas um cão apresentou hipercalemia. Não houve diferença estatística entre os momentos dentro do mesmo grupo $(P>0,05)$, contudo, foi observada diferença significativa entre os grupos no momento T4 (12h) $(P<0,05)$. Os cães do grupo envenenado apresentaram valores médios de potássio significativamente menores que os do grupo controle nesse momento $(\mathrm{P}<0,05)$, como demonstrado no Quadro 3.

Os níveis de cálcio iônico plasmático no grupo contro-

\begin{tabular}{|c|c|c|c|c|c|c|}
\hline \multirow[t]{2}{*}{ Grupos } & \multicolumn{6}{|c|}{ Momentos } \\
\hline & TO & $\mathrm{T} 1$ & $\mathrm{~T} 2$ & T3 & $\mathrm{T} 4$ & Valor P \\
\hline $\begin{array}{l}\text { Controle } \\
\text { Intoxicados } \\
\text { Valor P }\end{array}$ & $\begin{array}{c}0,96 \pm 0,11 \\
0,86 \pm 0,18 \\
(P=0,26)\end{array}$ & $\begin{array}{c}0,93 \pm 0,20 \\
0,74 \pm 0,28 \\
(P=0,19)\end{array}$ & $\begin{array}{c}0,97 \pm 0,06^{a} \\
0,74 \pm 0,22^{b} \\
(P=0,03)\end{array}$ & $\begin{array}{l}0,93 \pm 0,18 \\
0,74 \pm 0,20 \\
(P=0,074)\end{array}$ & $\begin{array}{c}0,98 \pm 0,08^{a} \\
0,74 \pm 0,23^{b} \\
(P=0,037)\end{array}$ & $\begin{array}{c}(P=0,969) \\
(P=0,416) \\
-\end{array}$ \\
\hline
\end{tabular}

Letras iguais na mesma coluna não diferem entre si $(P<0,05)$.

Quadro 3. Média e desvio padrão dos valores de potássio plasmático, em $\mathrm{mEq} / \mathrm{L}$, dos cães dos grupos controle $(n=5)$ e intoxicado com veneno de sapo $(n=15)$, nos diferentes momentos

\begin{tabular}{ccccccc}
\hline \multirow{2}{*}{ Grupos } & \multicolumn{7}{c}{ Momentos } \\
\cline { 2 - 7 } & T0 & T1 & T2 & T3 & T4 & Valor $P$ \\
\hline Controle & $3,3 \pm 0,26$ & $3,6 \pm 0,31$ & $3,4 \pm 0,41$ & $3,3 \pm 0,20$ & $3,9 \pm 0,40^{\mathrm{a}}$ & $(\mathrm{P}=0,082)$ \\
Intoxicados & $3,2 \pm 0,36$ & $3,4 \pm 0,50$ & $3,2 \pm 0,83$ & $3,1 \pm 0,65$ & $3,2 \pm 0,45^{\mathrm{b}}$ & $(\mathrm{P}=0,348)$ \\
Valor $\mathrm{P}$ & $(\mathrm{P}=0,40)$ & $(\mathrm{P}=0,45)$ & $(\mathrm{P}=0,53)$ & $(\mathrm{P}=0,44)$ & $(\mathrm{P}=0,008)$ & -
\end{tabular}

Letras iguais na mesma coluna não diferem entre si $(P<0,05)$. 
le variaram de $0,69 \mathrm{mg} / \mathrm{dL}$ a $1,17 \mathrm{mg} / \mathrm{dL}$, e todos os cães desse grupo apresentaram valores dentro da normalidade (DiBartola 2000). No grupo envenenado, os valores variaram de $0,31 \mathrm{mg} / \mathrm{dL}$ a $1,4 \mathrm{mg} / \mathrm{dL}$, e, da mesma maneira, os cães desse grupo apresentaram valores considerados normais para a espécie. Não se observou diferença significativa entre os momentos dentro do mesmo grupo $(P>0,05)$. Contudo, foi detectada diferença significativa entre os grupos nos momentos T2 (4h) e T4 (12h) $(P<0,05)$. Os cães envenenados apresentaram valores médios significativamente menores que os do grupo controle nestes dois momentos (Quadro 4).

\section{DISCUSSÃO}

Os cães do grupo envenenado experimentalmente com o veneno de sapo apresentaram valores de CK-MB significativamente maiores que os cães do grupo controle no momento T3. Esse resultado demonstra importância clínica, pois o aumento dessa enzima sugere agressão cardíaca, confirmando a cardiotoxicidade do veneno de sapo. Em relação aos valores de CK-MB encontrados nos cães envenenados, $60 \%$ estavam acima dos valores considerados normais para a espécie (Larsson 2005) no momento T3. Já com 24 horas após a administração do veneno (T5), todos os cães apresentaram valores dentro da faixa de normalidade. Esse fato confirma que a lesão é aguda, porém pode ser reversível, uma vez que os valores da enzima retornaram ao normal.

A Tnlc teve aumento significativo no momento T5. No momento T3, apesar de não haver diferença significativa, os valores de Tnlc obtidos em $47 \%$ dos cães do grupo envenenado estavam elevados se diretamente comparados com os valores normais (Larsson 2005). Os resultados obtidos possuem importância clínica, pois a elevação indica agressão ao cardiomiócitos e o aumento é diretamente proporcional à extensão da lesão induzida (Ricchiuti et al. 1998).

Os valores de sódio plasmático dos cães envenenados experimentalmente não sofreram alterações significativas, permanecendo dentro dos valores normais estabelecidos para cães (DiBartola 2000) em todos os momentos analisados. Alguns autores (Palumbo et al. 1975, Russel 1979, Peterson \& Roberts 2001) relatam a ocorrência de leve diminuição nos níveis de sódio plasmático em animais envenenados por veneno de sapo. Esses autores relatam que a alteração eletrolítica pode ser vista, pois, ao ocorrer o bloqueio da bomba de sódio e potássio pela ação da bufotoxina, o sódio se acumula no interior da célula. Esse fato, no entanto, não foi verificado neste estudo, possivelmente porque a troca entre os íons cálcio e sódio compensou o acúmulo inicial de sódio.

Em relação ao potássio, a hipocalemia observada, tanto nos cães controle quanto nos envenenados com bufotoxina, possivelmente se deve à fluidoterapia, porém a alteração foi mais evidente nos cães envenenados. $\mathrm{Na}$ comparação entre os grupos, a diferença significativa $(P<0,05)$ foi presenciada 12 horas após a administração do veneno (T4). Um cão envenenado apresentou hipercalemia. Esse achado é freqüentemente observado em cães e humanos envenenados com veneno de sapo, mas a hipocalemia também tem sido relatada (Eubig 2001).

Em relação ao cálcio iônico, os cães envenenados apresentaram valores significativamente menores que os dos cães controle nos momentos T2 e T4. Esse achado foi considerado sem significado clínico, uma vez que todos os valores estavam dentro dos parâmetros normais para cães (DiBartola 2000).

Alguns autores descrevem elevação dos níveis de potássio e cálcio plasmáticos em animais envenenados experimentalmente com bufotoxina (Palumbo et al. 1975, Russel 1979). A ocorrência de hipercalemia e a hipercalcemia justifica-se no envenenamento por bufotoxina, pois essa substância inibe a bomba de sódio e potássio ao se ligar à enzima Na-K-ATPase. O bloqueio da bomba provoca o acúmulo de potássio extracelular, eleva o sódio intracelular e, por estimular a troca entre sódio e cálcio, aumenta a concentração de cálcio nos cardiomiócitos (Palumbo \& Perry 1983, Chi et al. 1998, MacFarland 1999, Eubig 2001). Alguns autores (Chi et al. 1998) relatam uma correlação positiva significativa entre o nível sérico de potássio e a taxa de mortalidade em casos de envenenamento por veneno de sapo em humanos. Indivíduos com grave hipercalemia têm prognóstico desfavorável e necessitam de um tratamento mais intensivo.

\section{CONCLUSÃO}

Os cães envenenados experimentalmente por veneno de sapo (bufotoxina) apresentam elevação nos níveis dos marcadores cardíacos CK-MB e Tnlc, confirmando a cardiotoxicidade do veneno. As alterações eletrolíticas mais freqüentes foram a hipocalemia e hipocalcemia.

Agradecimentos.- À Fundação para o Desenvolvimento da Unesp (Fundunesp), pelo Auxílio Pesquisa.

\section{REFERÊNCIAS}

Bedford P.G.C. 1974. Toad venom toxicity and its clinical occurrence in small animals in the United Kingdom. Vet. Rec. 94:613-614.

Chen K.K. \& Kovarikova A. 1967. Pharmacology and toxicology of toad venom. Pharmacol. Exp. Ther. 56:1535-1541.

Chi H.T., Hung D.Z., Hu W.H. \& Yang D.Y. 1998. Prognostic implication of hiperkalemia in toad toxin intoxication. Human Exp. Toxicol. 17:343346.

Dibartola S.P. 2000. Fluid Therapy in Small Animal Practice. $2^{\text {nd }}$ ed. W.B. Saunders, Philadelphia, p.738-741.

Ettinger S.J. \& Feldman E.C. 1997. Tratado de Medicina Interna Veterinária. Vol.1. 4aㅡ ed. Manole, São Paulo, p.1382-1429.

Eubig P.A. 2001. Bufo species toxicosis: Big toad, big problem. Vet. Med. 96:595-599.

Germiniani H. 1978. Diagnóstico e terapêutica das arritmias cardíacas. $2^{\underline{a}}$ ed. Byk-Procienx, São Paulo, p.107-128.

Hoffman B.F. \& Lefkowitz R.J. 1991. Catecolaminas e drogas simpatomiméticas, p.123-144. In: Goodman L.S. \& Gilman A. (Eds), 
As Bases Farmacológicas da Terapêutica. $8^{\underline{a}}$ ed. Guanabara Koogan, Rio de Janeiro.

Knowles R.P. 1968. Toad poisoning in dogs. J. Am. Vet. Med. Assoc. 153:1202.

Larsson M.H., Santos A.L.F., Pereira G.G. \& Gutierrez V.C.R. 2005. Serum level of Creatine Kinase isoenzime MB and Cardiac Troponin I (CTnl) in dogs with normal electrocardiogram and in those with ST segment deviation using chemiluminescence assay. Proc. 30th World Congress of the World Small Animal Veterinary Association, Mexico City, Mexico, p.74. (Abstract)

MacDonald B. 1990. Terrier toad toxicity syndrome. Aust. Vet. Pract. 20:118.

McFarland P.J. 1999. Toad toxicity. Aust. Vet. Pract. 29:98-103.

Missov E., Calzolari C. \& Pau B. 1997. Circulation cardiac troponin I in severe congestive heart failure. Circulation 96:2953-2959.

Monti R. \& Cardelo L. 1994. Bioquímica do veneno de anfíbios, p.225232. In: Barraviera B. (Ed.), Venenos Animais: uma visão integrada. Editora de Publicações Científicas Ltda, Rio de Janeiro.

Morrison D.F. 1990. Multivariate Statistical Methods. McGrawl-Hill, São Paulo, p.311-314.

Klaassen C.D. 1996. Toxic effects of animal toxins, p.801-840. In: Casarett L.J. \& Doull J. (Eds), Toxicology: The basic science of poisons. $5^{\text {th }}$ ed. McGraw-Hill, New York.

Oliveira P.C.L. 1998. Uso de lidocaína, propranolol, verapamil e amiodarona na intoxicação por veneno de sapo (gênero Bufo) em cães. Dissertação de Mestrado, Faculdade de Medicina Veterinária e Zootecnia, Universidade Estadual Paulista, Botucatu, SP. 99p.

Otani A., Palumbo N.E. \& Read G. 1969. Pharmacodynamics and treatment of mammals poisoned by Bufo marinus toxin. Am. Vet. Res. 30:1865-1872.
Palumbo N.E., Perri S. \& Read G. 1975. Experimental induction and treatment of toad poisoning in the dog. J. Am. Vet. Med. Assoc. 167:1000-1005.

Palumbo N.E. \& Perry S.F. 1983. Toad poisoning, p.160-162. In: Kirk R.W. (Ed.), Current Veterinary Therapy: Small Animal Practice. $8^{\text {th }}$ ed. W.B. Saunders, Philadelphia.

Peterson M.E. \& Roberts B.K. 2001. Amphibian toxins, p.396-404. In: Peterson M.E. \& Talcott P.A. (Eds), Small Animal Toxicology. W.B. Saunders, Philadelphia.

Puschendorf B. 1999. Strategies for cardiac marker measurement. Clin. Chem. Lab. Med. 37:997-999.

Ricchiuti V., Sharkey S.W., Murakami M.M., Voss E.M. \& Apple F.S. 1998. Cardiac troponin I alterations in dog hearts with myocardial infarction. Am. J. Clin. Pathol. 110:241-247.

Russel F.E. 1979. Toad poisoning, p.183-185. In: Hoskins H.P. (Ed.), Canine Medicine. Vol.1. $4^{\text {th }}$ ed. American Veterinary Publications, Santa Barbara.

Sakate M. \& Oliveira P.C.L. 2000. Toad envenoming in dogs: Effects and treatment. J. Venom. Anim. Toxins 6:52-62.

Sakate M. \& Oliveira P.C.L. 2001. Use of lidocaine, propranolol, amiodarone and verapamil in toad envenoming (Genus Bufo) in dogs. J. Venom. Anim. Toxins 7:240-259.

Sonne L., Rozza D.B., Wolffenbutell A.N., Meirelles A.E.W., Pedroso P.M.O., Oliveira E.C. \& Driemeier D. 2008. Intoxicação por veneno de sapo em um canino. Ciência Rural 38:1787-1789.

Zar J.H. 1996. Biostatistical Analysis. Englewood Cliffs, Prentice Hall, p.16-52.

Zelnik R. 1965. A natureza química do veneno de sapo. Ciência e Cultura 17:10-14. 